\title{
P393
}

\section{Local Time Slowness Adaptive Filter and Optimal Window Strategies}

\author{
S. Ventosa* (UTM-CSIC), C. Simon (UTM-CSIC) \& M. Schimmel (IJA-CSIC)
}

\section{SUMMARY}

The local slant-stack transform (LSST) is a technique commonly used in seismic data processing to filter complex seismic waves, when the $\tau$-p transform and the f-k filters cannot be used. To automatically adapt the characteristics of the filters based on the LSST to the slowness variation of the wavefronts in seismic profiles these filters are usually combined with an instantaneous slowness estimator. To make an objective design, we analyze the relation between the slowness resolution, the window parameters and the main frequency components of the waves to employ the most suitable windows in order to achieve an optimum slowness and space resolutions. We further validate this design procedure with two synthetic examples. 


\section{Introduction}

One of the characteristics of any wave field is the similarity of the waveforms along the wavefront. This high lateral coherence can be used to estimate the instantaneous slowness (inverse of velocity) of each wave or even to separate the waves of interest in function of their slowness in order to analyse them with higher precision.

The local slant-stack transform (LSST) is a technique commonly used in seismic data processing to filter complex seismic waves using the lateral coherence, when the $\tau$ - $p$ transform and the $f-k$ pie-slice filters (Yilmaz and Doherty 2001) cannot be employed due to the lack of space resolution of the former and of time-space resolution of the latter. To automatically adapt the characteristics of LSST based filters to the slowness variation of the signals, these filters are usually combined with an instantaneous slowness estimators based on local coherence measures (Milkereit 1987, Duncan and Beresford 1994, Schimmel and Gallart 2003).

Up to date, the window of the LSST is designed empirically as a compromise between the slowness resolution and the wavefront tracking capability. The aim of this study is to provide some objective criteria to design the LSST window that achieves the optimum slowness and space resolutions.

\section{Window design criteria}

The continuous LSST can be written as:

$$
v_{s}\left(\tau, x_{c}\right)=\int_{-\infty}^{\infty} g\left(x-x_{c}\right) u\left(p_{s}\left(x-x_{c}\right)-\tau, x\right) d x
$$

where $u(t, x)$ is the original record section (RS), $v_{s}\left(\tau, x_{c}\right)$ the RS in the time-space-slowness domain and $g(x)$ a weighting function also called the space window.

If we define $u(t, x)=u(t-q x)$ as a profile of a seismic wave completely coherent along a line of slope $q$, it can be shown solving the above integral that the spectrum of $v_{s}\left(\tau, x_{c}\right)$ can be expressed as,

$$
\hat{v}_{s}\left(\omega, x_{c}\right)=\hat{u}(\omega) \hat{h}_{s}(\omega)=\hat{u}(\omega) \hat{g}\left(\left(q-p_{s}\right) \omega\right) e^{-j q x_{c} \omega}
$$

where $\hat{u}(\omega)$ is the waveform spectrum and $\hat{h}_{s}(\omega)$ a transfer function.

As can be seen in (2), the bandwidth of $\hat{h}_{s}(\omega)$ is inversely proportional to the factor $q-p_{s}$. Thus, if we define the frequency bandwidth of $\hat{g}(\omega)$ as $\Delta \omega$, the bandwidth of $\hat{h}_{s}(\omega)$ will be $\Delta \omega /\left|q-p_{s}\right|$. Therefore, if $\hat{u}(\omega)$ has a band-pass like spectrum, as the $p_{s}$ slowness moves away from the $q$ slowness of the planar wave $u(t-q x)$, their high frequency components are progressively attenuated until the whole wave is almost completely removed. In conclusion, the characteristics of the used window and the wavefront spectrum determine the slowness resolution of the LSST.

In the particular and common case of a pass-band signal $\hat{u}(\omega)$ that can be considered narrowband, the slowness bandwidth/resolution is equal to $\Delta p=\Delta \omega / \omega_{0}$. The minimum rejection in frequency is usually defined as the maximum amplitude of the sidelobes with respect to the main lobe,

$$
A=10 \log _{10} \frac{\left|\hat{g}\left(\left(q-p_{s}\right) \omega_{0}\right)\right|^{2}}{|\hat{g}(0)|^{2}}
$$

Although the sidelobe attenuation does not vary with the slowness, its central frequency will.

For example, in the case of a LSST that uses an L trace long rectangular window whose equivalent noise bandwidth is $\Delta \omega_{\mathrm{EN}}=1$, the equivalent noise slowness bandwidth is: 


$$
\Delta p=\frac{2 \pi \Delta \omega_{\mathrm{EN}}}{L \omega_{0}}=\frac{2 \pi}{L \omega_{0}}=\frac{1}{L f_{0}}
$$

and the minimum slowness rejection at the stopband, $A=-13 \mathrm{~dB}$.

\section{Filtering and synthesis}

Once we have filtered the RS in the time-space-slowness domain, we have to apply an inverse transform to synthesize the filtered RS back in the time-space domain. To perform this operation, we can follow two opposite strategies: synthesizing the filtered RS using only the components of interest, or, on the contrary, estimating the waves that we want to remove and subtract them from the original RS directly in the time-space domain.

However, as the energy of the waves of the RS is generally concentrated in a narrow slowness band, this operation can usually be approximated by the selection of the dominant instantaneous slowness component. This way the inverse operation becomes trivial, and the instability problems of the LSST disappear. Therefore, we will focus on two closely related applications: removing high energy interfering waves and estimating low energy waves.

The instantaneous slowness of the signals is estimated as the slowness component with the highest degree of coherence in a predefined slowness interval. To perform this measure we used the phase stack coherence estimators shown by Schimmel and Paulssen (1997) because they are amplitude unbiased and avoid the zero-crossing problems.

In the following, two synthetic examples will be shown. Some tests on real data have also been successfully performed but they won't be shown here due to lack of space. Apart from synthetic data, these filters have been applied on several real data examples, such as in wide-angle seismic profiles, where the refracted waves have been successfully separated from the background noise and other interfering signals. For the OBS data, the direct high-energy water reverberations have also been attenuated causing minimal distortions to the other signals such as the crustal low-energy reflected/refracted waves under study.

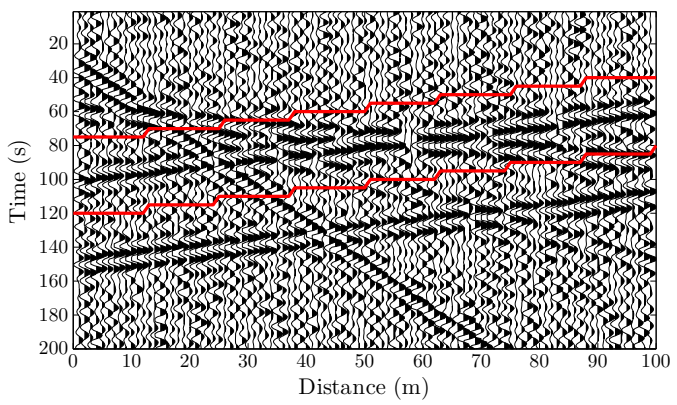

Figure 1 Synthetic RS composed of five plane waves, the red lines specify the work area.

\section{Example: five seismic plane waves}

The RS shown in figure 1 is composed by five planar waves with a Gaussian envelope and a Gaussian noise of power of 0.1 with the same amplitude spectrum as the waves. Their central frequency is $1 / 9$ $\mathrm{Hz}$ and their amplitude equals one, except for the $1 \mathrm{~s} / \mathrm{m}$ wave that is $30 \%$ smaller. In this example, we want to attenuate the wave with slowness $-0.4 \mathrm{~s} / \mathrm{m}$ and pic time at distance $0 \mathrm{~m}$ at $100 \mathrm{~s}$-delimited in red- without distorting the other waves. The noise attenuation has not been the objective of this test. 


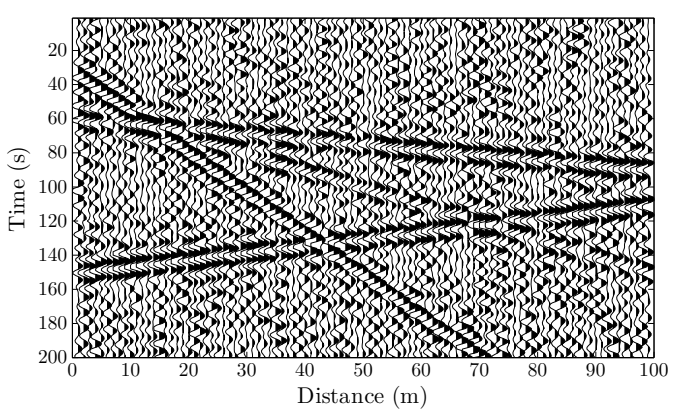

(a) Filtered section with a 27 m long window.

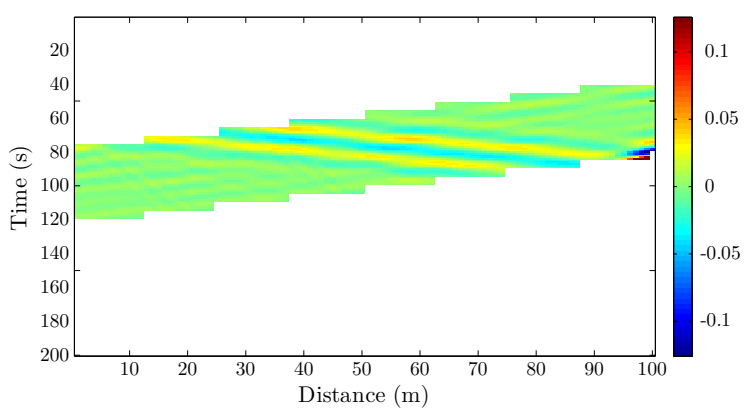

(c) Amplitude mean error of (a).

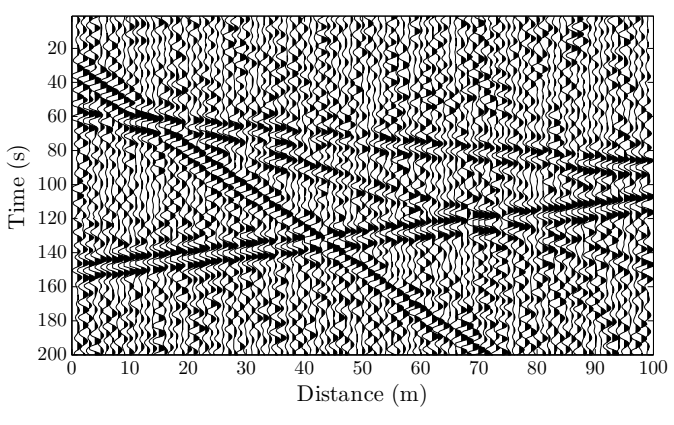

(b) Filtered section with a 17 m long window.

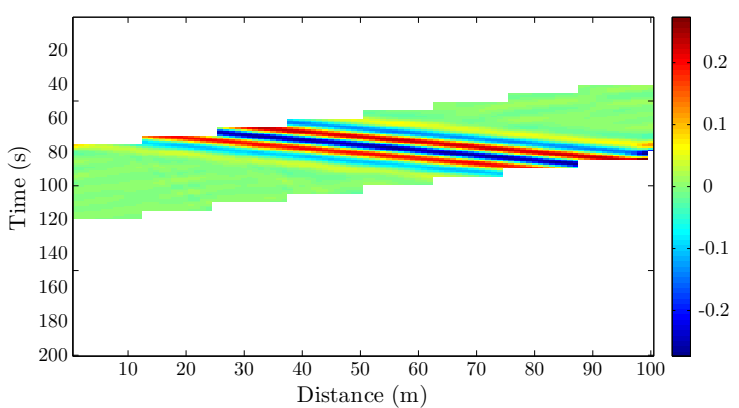

(d) Amplitude mean error of (c).

Figure 2 Filtered RS with a non-adaptive LSST based filter to remove the wave delimited in red in figure 1 and their mean errors. (a) and (b) use the same normalization as the one in figure 1 .

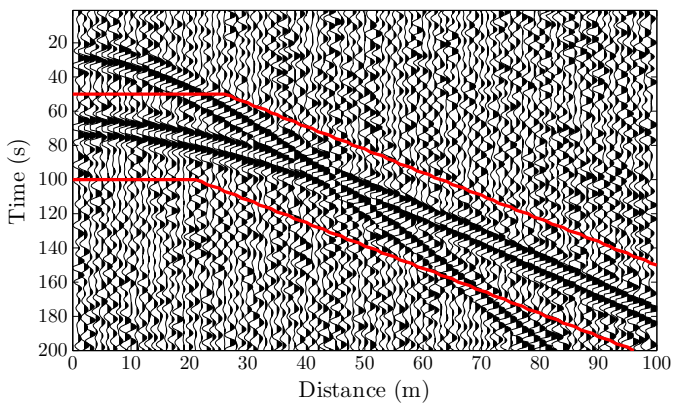

(a) Original $R S$.

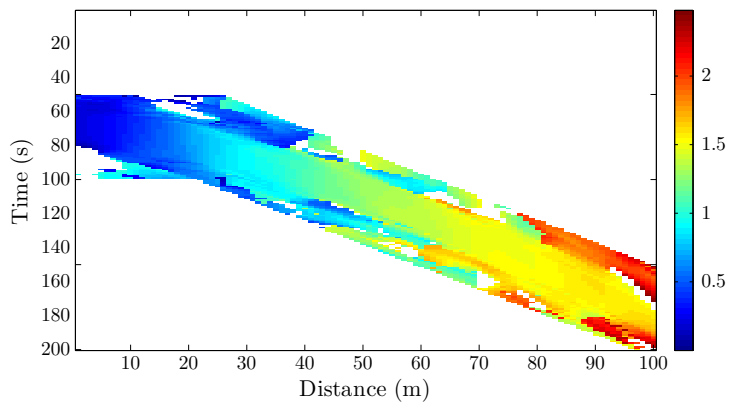

(c) Slowness measure $(\mathrm{s} / \mathrm{m})$.

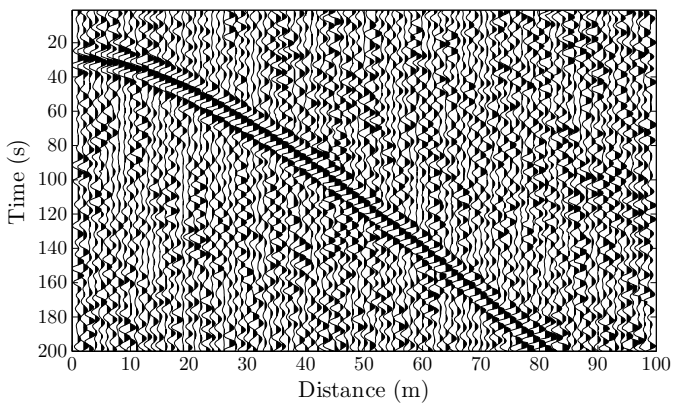

(b) Filtered RS.

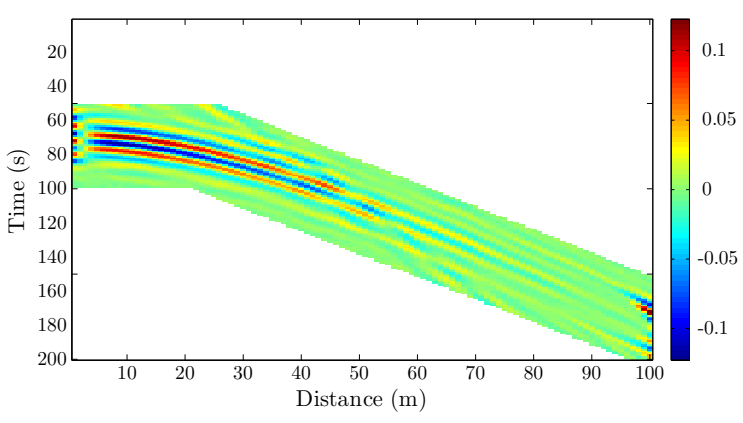

(d) Amplitude mean error.

Figure 3 Synthetic RS composed of two waves with a variable wavefront trajectories filtered with an adaptive LSST based filter. 
Given a particular window, its minimum length is fixed by the minimum resolution required to distinguish the wave to estimate from the other ones. Since the closest wave in slowness is at $0.3 \mathrm{~s} / \mathrm{m}$, the minimum resolution will be $\Delta p_{\min }=0.7 \mathrm{~s} / \mathrm{m}$; therefore, by means of (4) the minimum length should be $L \geq 12.86 \Delta \omega_{\mathrm{N}} \mathrm{m}$ where $\Delta \omega_{\mathrm{N}}$ is the bandwidth. In this example, we have chosen a Hamming window; if we approximate its bandwidth by half of the bandwidth $\Delta \omega_{\mathrm{z}}$ between zeros, $\Delta \omega_{\mathrm{N}}=\Delta \omega_{\mathrm{z}} / 2=2$, the minimum length to have an attenuation at $0.3 \mathrm{~s} / \mathrm{m}$ approximately equal to the stopband attenuation should be $27 \mathrm{~m}$.

When a $27 \mathrm{~m}$ long window is used, figures 2(a) and (c), we can notice that the interfering signal has been removed without an appreciable distortion of the other waves. But, can be seen in figures 2 (b) and (d), the reduction of the slowness resolution below the minimum required causes a severe reduction in the rejection level of the closest wave.

\section{Example: Two seismic waves of variable slowness}

The RS shown in figure 3(a) consists of two waves with a hyperbolic wavefront trajectory, and a Gaussian noise of power 0.05 . Their central frequency is $1 / 9 \mathrm{~Hz}$ and their amplitude is one. In this example, we want to attenuate the second wave-delimited in red-without distorting the other one.

Just before the intersection point between the two waves, approximately at $30 \mathrm{~m}$, is the point where the waves overlap with the minimal slowness difference which, according to (4), implies a minimum length of $L \geq 8.18 \Delta \omega_{\mathrm{N}} \mathrm{m}$. In figure 3(b), we can see the filtered RS with a $17 \mathrm{~m}$ long Hamming window together with their mean errors. To measure the slowness, figure $3(\mathrm{c})$, we have used a phase stack coherence estimators with a $23 \mathrm{~m}$ long Hamming window.

Finally, it is important to remember that the main assumption made in the LSST is that the coherent waves suffer mild slowness variations along their wavefront trajectories. However, when these variations are no longer negligible for the window length used, an important error in the estimation of the waves appears, as we can notice in figure 3(d) between 0 and $40 \mathrm{~m}$.

\section{Conclusions}

The adaptive LSST based filters are a powerful tool that enables an easy separation of the seismic waves without having accurate measurements of their wavefront trajectories, with a good degree of control of the slowness resolution, when commonly used techniques such as the pie-slice f-k filters or the $\tau$-p transform cannot be applied. The design of the LSST window entails a compromise between the slowness resolution and the wavefront tracking capability. To objectively solve this problem and achieve an optimum window, we have calculated the minimum window length required for a given window shape to achieve the minimum slowness resolution to estimate a seismic wave thereby minimizing the interference caused by the other waves.

\section{References}

Duncan, G. and Beresford, G. [1994], Slowness adaptive f-k filtering of prestack seismic data, Geophysics, 59(1), $140-147$.

Milkereit, B. [1987], Decomposition and inversion of seismic data -an instantaneous slowness approach, Geophysical Prospecting, 35, 875-894.

Schimmel, M. and Gallart, J. [2003\}, The use of instantaneous polarization attributes for seismic signal detection and image enhancement, Geophysical Journal International, 155(2), 653-668.

Schimmel, M. and Paulssen, H. [1997], Noise reduction and detection of weak coherent signals through phase-weighted stacks, Geophysical Journal International, 130(2), 497-505.

Yilmaz, O. and Doherty, S. M. [2001], Seismic data analysis: processing, inversion and interpretation of seismic data, Society of exploration geophysicists. 